\title{
Finite-Time Projective Synchronization of Caputo Type Fractional Complex-Valued Delayed Neural Networks
}

\author{
Shuang Wang ${ }^{1}$, Hai Zhang ${ }^{1, *(\mathbb{D}) \text {, Weiwei Zhang }}{ }^{1,2}{ }^{\mathbb{D}}$ and Hongmei Zhang ${ }^{1}$ \\ 1 School of Mathematics and Physics, Anqing Normal University, Anqing 246133, China; \\ wangshuang@aqnu.edu.cn (S.W.); zhangweiwei@aqnu.edu.cn (W.Z.); zhanghongmei@aqnu.edu.cn (H.Z.) \\ 2 Department of Mathematics, Nanjing University of Aeronautics and Astronautics, Nanjing 211106, China \\ * Correspondence: zhanghai@aqnu.edu.cn
}

Citation: Wang, S.; Zhang, H.;

Zhang, W.; Zhang, H. Finite-Time

Projective Synchronization of Caputo

Type Fractional Complex-Valued

Delayed Neural Networks.

Mathematics 2021, 9, 1406.

https://doi.org/10.3390/math

9121406

Academic Editor: Ivan Matychyn

Received: 11 May 2021

Accepted: 14 June 2021

Published: 17 June 2021

Publisher's Note: MDPI stays neutral with regard to jurisdictional claims in published maps and institutional affiliations.

Copyright: (C) 2021 by the authors. Licensee MDPI, Basel, Switzerland. This article is an open access article distributed under the terms and conditions of the Creative Commons Attribution (CC BY) license (https:/ / creativecommons.org/licenses/by/ $4.0 /)$.

\begin{abstract}
This paper focuses on investigating the finite-time projective synchronization of Caputo type fractional-order complex-valued neural networks with time delay (FOCVNNTD). Based on the properties of fractional calculus and various inequality techniques, by constructing suitable the Lyapunov function and designing two new types controllers, i.e., feedback controller and adaptive controller, two sufficient criteria are derived to ensure the projective finite-time synchronization between drive and response systems, and the synchronization time can effectively be estimated. Finally, two numerical examples are presented to verify the effectiveness and feasibility of the proposed results.
\end{abstract}

Keywords: fractional-order complex-valued neural networks; finite-time projective synchronization; feedback control; adaptive control

\section{Introduction}

As a branch of mathematical analysis, fractional calculus mainly deals with differential or integration of arbitrary non-integer order. Fractional calculus is especially suitable for describing the physical change process with memory property and historical dependence, and most of the research objects in the actual system have such properties, which are also main advantages. Compared with the integer-order derivative model, their differences are mainly reflected in the following aspects: in time, the integer-order differential equation represents a change or a certain property of a physical or mechanical process at a certain moment, which cannot describe the memory characteristics of neurons, while the fractional derivative contains all the information from the start time to the present time, so that it can describe the memory property of neurons more truly. With the deepening of the theoretical research of fractional calculus, fractional calculus has been widely used in various fields.

Recently, researchers have found that it was excellent to introduce fractional calculus theory into the research of neural networks. Since fractional calculus has infinite memory, some scholars have turned it into neural networks and established fractional-order neural network models, it can better describe the dynamic behaviors of neurons. Since there are many types of input variables, state variables, activation functions, and connection weights, neural networks can be divided into two categories: real-valued neural networks (RVNNs) and complex-valued neural networks (CVNNs). However, the CVNNs completes the information processing in the complex number domain, which is still very different from the RVNNs. It should be pointed that CVNNs has advantage in solving some difficult real-world problems [1-6].

As a typical phenomenon, synchronization is widely concerned because of its application in signal processing [7], image encryption [8], and pattern recognition [9]. So far, several types of synchronization have been studied, such as complete synchronization [10], lag projective synchronization [11], antisynchronization [12], quasiuniform synchronization [13], projective synchronization [14], global asymptotical synchronization [15], and 
global Mittag-Leffler synchronization [16,17]. Compared with the above synchronization types, projective synchronization can achieve faster communication due to its proportional characteristics. Recently, in order to realize the synchronization of two chaotic systems, many synchronization strategies have been designed, including adaptive control [18,19], pulse control [20], intermittent control [21], and feedback control [22,23]. It shows that an appropriate controller can synchronize the response system with the drive system at an infinite level. Self-adaptation is a feature that the research object can change its own habits to adapt to the new environment. Adaptive control is an unpredictable change of the system, which can make the system adjust its own characteristics according to the environmental changes to achieve the desired optimal state. According to the obtained process information and a certain design method, the adaptive control system makes control decisions to modify the structure, parameters or control function of the controller, so that the control effect is optimal or approximately better.

Based on the above discussion, under the premise that the complex-valued system is regarded as a whole, this paper mainly studies the finite-time projective synchronization problem of a class of FOCVNNTD. Firstly, based on the finite-time projective synchronization theory, two different types of controllers are introduced. Then by constructing the Lyapunov function, using feedback controller and adaptive controller, some new sufficient conditions for the finite-time projective synchronization of the considered FOCVNNTD are given. Furthermore, the synchronization time is effectively estimated, which is related to the order of the fractional derivative and the control parameters. Finally, the theoretical results in this thesis are verified by numerical simulations.

The main structure of this thesis: In the Section 2, the definition of fractional derivative and several important lemmas are presented. In the Section 3, by using a feedback controller and adaptive controller, some different synchronization criteria are established. The fourth part gives numerical exercises to demonstrate the effectiveness and applicability of the obtained results. Finally, the direction of future work is pointed out.

\section{Preliminaries and Model Description}

In this section, we first review some basic knowledge about the fractional calculus. Then several key lemmas and requisite assumptions are introduced for the following discussion. Finally, we establish a FOCVNNTD model.

Definition 1 ([24]). The Caputo fractional derivative of order $\mu$ for a function $h(t) \in C^{n+1}([0,+\infty), R)$ is defined as:

$$
t_{0} D_{t}^{\mu} h(t)=\frac{1}{\Gamma(n-\mu)} \int_{t_{0}}^{t}(t-\tau)^{n-\mu-1} h^{(n)}(\tau) d \tau,
$$

and $t>t_{0}, n-1<\mu<n \in Z^{+}, \Gamma(\cdot)$ is the Gamma function, where

$$
\Gamma(\mu)=\int_{0}^{\infty} t^{\mu-1} e^{-t} d t
$$

Particularly, when $0<\mu<1$,

$$
{ }_{t_{0}} D_{t}{ }^{\mu} h(t)=\frac{1}{\Gamma(1-\mu)} \int_{t_{0}}^{t}(t-\tau)^{-\alpha} h^{\prime}(\tau) d \tau
$$

Lemma 1 ([25]). Let $h(t) \in C^{n}[0,+\infty),{ }_{0} D_{t}^{-\mu} h(t)$ is the Riemann-Liouville fractional integral, where $n-1<\mu, v<n \in Z^{+}$,

$$
\begin{aligned}
& \text { (1) }{ }_{0} D_{t}^{-\mu}{ }_{0} D_{t}^{-v} h(t)={ }_{0} D_{t}^{-(\mu+v)} h(t), \mu, v \geq 0 ; \\
& \text { (2) }{ }_{0} D_{t}^{-\mu}{ }_{0} D_{t}^{-v} h(t), \mu=v \geq 0 ; \\
& \text { (3) }{ }_{0} D_{t}^{-\mu}{ }_{0} D_{t}^{v} h(t)=h(t)-\sum_{k=0}^{n-1} \frac{t^{k}}{k !} h^{(k)}(0), \mu=v \geq 0 .
\end{aligned}
$$


Lemma 2 ([26]). Let $h(t) \in C^{n}[0,+\infty)$ is continuous and analytic function, the following inequality holds:

$$
{ }_{t_{0}}^{C} D_{t}^{\mu} h(t) \overline{h(t)} \leq h(t)_{t_{0}}^{C} D_{t}^{\mu} \overline{h(t)}+\overline{h(t)} t_{t_{0}}^{C} D_{t}^{\mu} h(t),
$$

and $t \geq t_{0}, 0<\mu<1$.

Lemma 3 ([27]). If $x$ and $y$ are any two complex numbers, and for any real constant $\rho$, then the inequality is established as follows:

$$
x \bar{y}+\bar{x} y \leq \rho x \bar{x}+\frac{1}{\rho} y \bar{y}
$$

Lemma 4 ([28]). Suppose that function $h(t) \in C^{n}[0,+\infty)$ is continuous and differentiable on $t \in\left[t_{0}, \infty\right)$, then for any real constant $\omega$, it has

$$
{ }_{t_{0}}^{C} D_{t}^{\mu}(h(t)-\omega)^{2} \leq 2(h(t)-\omega)_{t_{0}}^{C} D_{t}^{\mu} h(t) .
$$

Lemma 5 ([29]). Let $m \in N, x_{1}, x_{2}, \cdots, x_{m}$ are non-negative real numbers, then for $m>1$,

$$
\left(\sum_{k=1}^{n} x_{k}\right)^{m} \leq n^{m-1} \sum_{k=1}^{n} x_{k}^{m}
$$

Lemma 6 ([30]). Assume that function $H(t)$ is continuous, positive-definite, and satisfies the following inequality,

$$
{ }_{t_{0}}^{C} D_{t}^{\mu} H(t) \leq-d H^{\gamma}(t)
$$

where $d>0,0<\gamma<\mu$ and all of them are constants. Then $H(t)$ satisfies the inequality as follows:

$$
H^{\mu-\gamma}(t) \leq H^{\mu-\gamma}\left(t_{0}\right)-\frac{d \Gamma(1+\mu-\gamma)\left(t-t_{0}\right)^{\mu}}{\Gamma(1+\mu) \Gamma(1-\gamma)}, t_{0} \leq t \leq t_{1}
$$

and $\lim _{t \rightarrow \infty} H(t)=0$, for all $t \geq T$, here $T$ is expressed by

$$
T=t_{0}+\left(\frac{\Gamma(1+\mu) \Gamma(1-\gamma) H^{\mu-\gamma}\left(t_{0}\right)}{d \Gamma(1+\mu-\gamma)}\right)^{\frac{1}{\mu}}
$$

Consider a class of FOCVNNTD as the drive system:

$$
\left\{\begin{array}{l}
D^{\mu} x_{k}(t)=-c_{k} x_{k}(t)+\sum_{j=1}^{n} a_{k j} f_{j}\left(x_{j}(t)\right)+\sum_{j=1}^{n} b_{k j} g_{j}\left(x_{j}(t-\tau)\right)+I_{k} \\
x_{k}(s)=\varphi_{k}(s), s \in\left[t_{0}-\tau, t_{0}\right]
\end{array},\right.
$$

where $D^{\mu}$ is the Caputo derivative, $k, j \in N=(1,2, \cdots, n), 0<\mu<1, n$ corresponds to the number of units; $x_{k}(t) \in C$ denotes the state variable of the drives system in time $t$, $f_{j}(\cdot), g_{j}(\cdot) \in C \rightarrow C$ represent the activation function without time delay and with time delay, respectively. $a_{k j}, b_{k j} \in C$ stand for the connection weight and the delay connection weight, respectively, $c_{k}>0$ is the self-regulating parameters; $\tau>0$ is time delay; and $I_{k}$ denotes the external input. $\varphi_{k}(t)$ is real-valued continuous function on $[-\tau, 0]$, its norm is given by $\|\varphi\|=\sup _{t \in[-\tau, 0]} \sum_{k=1}^{n}\left|\varphi_{k}(t)\right|$. 
In order to study the finite-time projective synchronization of the FOCVNNTD, the corresponding response system is defined by:

$$
\left\{\begin{array}{l}
D^{\mu} y_{k}(t)=-c_{k} y_{k}(t)+\sum_{j=1}^{n} a_{k j} f_{j}\left(y_{j}(t)\right)+\sum_{j=1}^{n} b_{k j} g_{j}\left(y_{j}(t-\tau)\right)+I_{k}+u_{k}(t) \\
y_{k}(s)=\phi_{k}(s), s \in\left[t_{0}-\tau, t_{0}\right]
\end{array},\right.
$$

$y_{k}(t)$ is the state variable of the response system and $u_{k}(t)$ denotes appropriate controller. For the sake of the main results can be obtained, an assumption is given below:

Assumption 1. Assume that $f(\cdot)$ and $g(\cdot)$ satisfy the Lipschitz condition, for any $x, y \in R$, there exists two real number $F_{j}$ and $G_{j}(j=1,2, \cdots, n)$, such that $\left|f_{j}(x)-f_{j}(y)\right| \leq F_{j}|x-y|$, $\left|g_{j}(x)-g_{j}(y)\right| \leq G_{j}|x-y|$.

Definition 2 ([31]). Under the appropriate controller, there exist numbers $\left\{t_{0}, J, \delta, \varepsilon\right\}, \delta<\varepsilon$, if and only if, for $\forall t \in J=\left[t_{0}, t_{0}+T\right]$, there exists a small error bound $\varepsilon>0$ such that $\|\boldsymbol{e}(t)\|<\varepsilon$, then the drive system (8) and the response system (9) can realize finite-time projective synchronization, where $t_{0}$ is the initial observation time.

\section{Results}

In this section, we will discuss the finite-time projective synchronization of FOCVNNTD by designing feedback controller and adaptive controller. Define the error system $e_{k}(t)=y_{k}(t)-\beta x_{k}(t)$ between drive system (8) and response system (9), where $\beta$ is the projective coefficient. Then

$$
\begin{aligned}
& D^{\mu} e_{k}(t)=-c_{k} e_{k}(t)+\sum_{j=1}^{n} a_{k j}\left[f_{j}\left(y_{j}(t)\right)-\beta f_{j}\left(x_{j}(t)\right)\right] \\
& +\sum_{j=1}^{n} b_{k j}\left[g_{j}\left(y_{j}(t-\tau)\right)-\beta f_{j}\left(x_{j}(t-\tau)\right)\right]+(1-\beta) I_{k}+u_{k}(t)
\end{aligned}
$$

\subsection{Finite-Time Projective Synchronization with Feedback Controller}

To achieve the finite-time projective synchronization between drive system (8) and response system (9), select the feedback controller as follows:

$$
\left\{\begin{aligned}
u_{k}(t)= & \pi_{k}(t)+w_{k}(t) \\
\pi_{k}(t)= & \sum_{j=1}^{n} a_{k j}\left[\beta f_{j}\left(x_{j}(t)\right)-f_{j}\left(\beta x_{j}(t)\right)\right] \\
& \quad+\sum_{j=1}^{n} b_{k j}\left[\beta g_{j}\left(x_{j}(t-\tau)\right)-f_{j}\left(\beta x_{j}(t-\tau)\right)\right]+(\beta-1) I_{k} \\
w_{k}(t)= & -\eta_{k} e_{k}(t)-\frac{\lambda_{k} e_{k}(t)}{\left(e_{k}(t) \overline{\left.e_{k}(t)\right)^{q}}\right.}
\end{aligned}\right.
$$

where $q \in(1-\mu, 1), \eta_{k}, \gamma_{k} \in C, \eta_{k}^{R}$, and $\lambda_{k}^{R}$ are all real numbers.

Theorem 1. On the premise of assumption 1 and the feedback controller (11), if the following conditions hold

$$
P>n \zeta, \zeta>1,
$$

then drive system (8) and response system (9) can achieve the finite-time projective synchronization, where

$$
P=\min _{1 \leq k \leq n}\left[\left(c_{k}+\bar{c}_{k}+\eta_{k}+\bar{\eta}_{k}\right)-\sum_{j=1}^{n}\left(a_{k j} \bar{a}_{k j} F_{j} \bar{F}_{j}+b_{k j} \bar{b}_{k j} G_{j} \bar{G}_{j}\right)-n\right] .
$$


Proof of Theorem 1. Based on the feedback controller (11), by using Lipschtz condition, one has

$$
\begin{gathered}
D^{\mu} e_{k}(t)=-\left(c_{k}+\eta_{k}\right) e_{k}(t)+\sum_{j=1}^{n} a_{k j}\left[f_{j}\left(y_{j}(t)\right)-f_{j}\left(\beta x_{j}(t)\right)\right] \\
+\sum_{j=1}^{n} b_{k j}\left[g_{j}\left(y_{j}(t-\tau)\right)-g_{j}\left(\beta x_{j}(t-\tau)\right)\right]-\frac{\lambda_{k} e_{k}(t)}{\left(e_{k}(t) \overline{\left.e_{k}(t)\right)^{q}}\right.} \\
\leq-\left(c_{k}+\eta_{k}\right) e_{k}(t)+\sum_{j=1}^{n} a_{k j} F_{j} e_{j}(t)+\sum_{j=1}^{n} b_{k j} G_{j} e_{j}(t-\tau)-\frac{\lambda_{k} e_{k}(t)}{\left(e_{k}(t) \bar{e}_{k}(t)\right)^{q}},
\end{gathered}
$$

The Lyapunov function is chosen as follows:

$$
V_{1}(t)=\sum_{k=1}^{n} e_{k}(t) \overline{e_{k}(t)}
$$

Using Lemmas 1-5, according to Equation (10), we obtain

$$
\begin{aligned}
& D^{\mu} V_{1}(t) \leq \sum_{k=1}^{n}\left(e_{k}(t) D^{\mu} \overline{e_{k}(t)}+\overline{e_{k}(t)} D^{\mu} e_{k}(t)\right) \\
& =\sum_{k=1}^{n}\left[-\left(c_{k}+\overline{c_{k}}+\eta_{k}+\overline{\eta_{k}}\right) e_{k}(t) \overline{e_{k}(t)}+\sum_{j=1}^{n}\left(a_{k j} \overline{F_{j}} \overline{e_{k}(t)} e_{j}(t)+\overline{a_{k j}} \overline{F_{j}} e_{k}(t) \overline{e_{j}(t)}\right)\right. \\
& \left.+\sum_{j=1}^{n}\left(b_{k j} G_{j} \overline{e_{k}(t)} e_{j}(t-\tau)+\overline{b_{k j} G_{j}} e_{k}(t) \overline{e_{j}(t-\tau)}\right)-\left(\lambda_{k}+\overline{\lambda_{k}}\right)\left(e_{k}(t) \overline{e_{k}(t)}\right)^{1-q}\right] \\
& \leq \sum_{k=1}^{n}\left[e_{k}(t) \cdot\left(-\left(\bar{c}_{k}+\bar{\eta}_{k}\right) \overline{e_{k}(t)}+\sum_{j=1}^{n} \bar{a}_{k j} \bar{F}_{j} \overline{e_{j}(t)}+\sum_{j=1}^{n} \bar{b}_{k j} \overline{G_{j}} \overline{e_{j}(t-\tau)}-\frac{\overline{\lambda_{k}} \cdot \overline{e_{k}(t)}}{\left(e_{k}(t) \overline{\left.e_{k}(t)\right)^{q}}\right.}\right)\right. \\
& \left.+\overline{e_{k}(t)}\left(-\left(c_{k}+\eta_{k}\right) e_{k}(t)+\sum_{j=1}^{n} a_{k j} F_{j} e_{j}(t)+\sum_{j=1}^{n} b_{k j} G_{j} e_{j}(t-\tau)-\frac{\lambda_{k} e_{k}(t)}{\left(e_{k}(t) \overline{\left.e_{k}(t)\right)^{q}}\right.}\right)\right] \\
& \leq \sum_{k=1}^{n}\left[-\left(c_{k}+\overline{c_{k}}+\eta_{k}+\overline{\eta_{k}}\right) e_{k}(t) \overline{e_{k}(t)}+\sum_{j=1}^{n}\left(a_{k j} \overline{a_{k j}} F_{j} \overline{F_{j}} e_{k}(t) \overline{e_{k}(t)}+e_{j}(t) \overline{e_{j}(t)}\right)\right. \\
& \left.+\sum_{j=1}^{n}\left(b_{k j} \overline{b_{k j}} G_{j} \overline{G_{j}} e_{k}(t) \overline{e_{k}(t)}+e_{j}(t-\tau) \overline{e_{j}(t-\tau)}\right)-\left(\lambda_{k}+\overline{\lambda_{k}}\right)\left(e_{k}(t) \overline{e_{k}(t)}\right)^{1-q}\right] \\
& =-\sum_{k=1}^{n}\left[\left(c_{k}+\overline{c_{k}}+\eta_{k}+\overline{\eta_{k}}\right)-\sum_{j=1}^{n}\left(a_{k j} \overline{a_{k j}} F_{j} \overline{F_{j}}+b_{k j} \overline{b_{k j}} G_{j} \overline{G_{j}}\right)\right] e_{k}(t) \overline{e_{k}(t)} \\
& +\sum_{k, j=1}^{n}\left(e_{j}(t) \overline{e_{j}(t)}+e_{j}(t-\tau) \overline{e_{j}(t-\tau)}\right)-\sum_{k=1}^{n}\left(\lambda_{k}+\overline{\lambda_{k}}\right)\left(e_{k}(t) \overline{e_{k}(t)}\right)^{1-q} \\
& \leq-\sum_{k=1}^{n}\left[\left(c_{k}+\overline{c_{k}}+\eta_{k}+\overline{\eta_{k}}\right)-\sum_{j=1}^{n}\left(a_{k j} \overline{a_{k j}} F_{j} \overline{F_{j}}+b_{k j} \overline{b_{k j}} G_{j} \overline{G_{j}}\right)\right] e_{k}(t) \overline{e_{k}(t)} \\
& +n \sum_{k=1}^{n}\left(e_{k}(t) \overline{e_{k}(t)}+e_{k}(t-\tau) \overline{e_{k}(t-\tau)}\right)-\sum_{k=1}^{n}\left(\lambda_{k}+\overline{\lambda_{k}}\right)\left(e_{k}(t) \overline{e_{k}(t)}\right)^{1-q} \\
& =-\sum_{k=1}^{n}\left[\left(c_{k}+\overline{c_{k}}+\eta_{k}+\overline{\eta_{k}}\right)-\sum_{j=1}^{n}\left(a_{k j} \overline{a_{k j}} F_{j} \overline{F_{j}}+b_{k j} \overline{b_{k j}} G_{j} \overline{G_{j}}\right)-n\right] e_{k}(t) \overline{e_{k}(t)} \\
& +n \sum_{k=1}^{n} e_{k}(t-\tau) \overline{e_{k}(t-\tau)}-\sum_{k=1}^{n}\left(\lambda_{k}+\overline{\lambda_{k}}\right)\left(e_{k}(t) \overline{e_{k}(t)}\right)^{1-q} \\
& \leq-P V_{1}(t)+n V_{1}(t-\tau)-d V_{1}^{1-q}(t) \\
& \leq-(P-n \zeta) V_{1}(t)-d V_{1}^{1-q}(t)
\end{aligned}
$$

for $\zeta>1, d=\min _{1 \leq k \leq n}\left(\lambda_{k}+\overline{\lambda_{k}}\right)$, if $P>n \zeta$,

$$
P=\min _{1 \leq k \leq n}\left[\left(c_{k}+\overline{c_{k}}+\eta_{k}+\overline{\eta_{k}}\right)-\sum_{j=1}^{n}\left(a_{k j} \overline{a_{k j}} F_{j} \overline{F_{j}}+b_{k j} \overline{b_{k j}} G_{j} \overline{G_{j}}\right)-n\right],
$$


according to Lemma 6, one can get

$$
D^{\mu} V_{1}(t) \leq-d V_{1}^{1-q}(t)
$$

then

$$
V_{1}^{(\mu+q-1)}(t) \leq V_{1}^{(\mu+q-1)}(0)-\frac{d \Gamma(\mu+q) t^{\mu}}{\Gamma(1+\mu) \Gamma(q)}, 0 \leq t \leq t_{1}
$$

for $t \geq t_{1}$, we have $V(t)=0$, here $t_{1}$ can be estimated by

$$
t_{1}=\left(\frac{\Gamma(1+\mu) \Gamma(q) V_{1}^{\mu+q-1}(0)}{d \Gamma(\mu+q)}\right)^{\frac{1}{\mu}} .
$$

So when $t \geq t_{1}$, we have $\lim _{t \rightarrow \infty}\left\|e_{k}(t)\right\|=0$, then system (8) and system (9) will reach the finite-time projective synchronization under the feedback controller (11).

\subsection{Finite-Time Projective Synchronization with Adaptive Controller}

To achieve the finite-time projective synchronization between the drive system (8) and response system (9), we can design the following adaptive controller

$$
\left\{\begin{array}{rl}
u_{k}(t)= & \pi_{k}(t)+v_{k}(t) \\
\pi_{k}(t)= & \sum_{j=1}^{n} a_{k j}\left[\beta f_{j}\left(x_{j}(t)\right)-f_{j}\left(\beta x_{j}(t)\right)\right] \\
& \quad+\sum_{j=1}^{n} b_{k j}\left[\beta g_{j}\left(x_{j}(t-\tau)\right)-f_{j}\left(\beta x_{j}(t-\tau)\right)\right]+(\beta-1) I_{k}
\end{array},\right.
$$

where

$$
\mu \in\left(\frac{1}{2}, 1\right), q \in\left(1-\mu, \frac{1}{2}\right), D^{\mu} \eta_{k}(t)=e_{k}(t) \overline{e_{k}(t)}-\sigma_{k} \operatorname{sign}\left(\eta_{k}(t)-\widetilde{\eta}_{k}\right)\left|\eta_{k}(t)-\bar{\eta}_{k}\right|^{1-2 q} .
$$

In the controller $(14), \eta_{k}(t)$ is determined by the input, state, output or performance parameters of the system.

Theorem 2. On the premise of assumption 1 and the adaptive controller (14), if the following conditions are satisfied

$$
Q>n \zeta^{\prime}, \zeta^{\prime}>1
$$

then the finite-time projective synchronization between the system (8) and the system (9) will be completed, where

$$
Q=\min _{1 \leq k \leq n}\left[\left(c_{k}+\bar{c}_{k}+2 \widetilde{\eta}_{k}\right)-\sum_{j=1}^{n}\left(a_{k j} \overline{a_{k j}} F_{j} \overline{F_{j}}+b_{k j} \overline{b_{k j}} G_{j} \overline{G_{j}}\right)-n\right] .
$$

Proof of Theorem 2. Based on the adaptive controller (14) and Lipschtz condition, we have

$$
\begin{aligned}
& D^{\mu} e_{k}(t)=-c_{k} e_{k}(t)+\sum_{j=1}^{n} a_{k j}\left[f_{j}\left(y_{j}(t)\right)-f_{j}\left(\beta x_{j}(t)\right)\right] \\
& +\sum_{j=1}^{n} b_{k j}\left[g_{j}\left(y_{j}(t-\tau)\right)-g_{j}\left(\beta x_{j}(t-\tau)\right)\right]-\eta_{k}(t) e_{k}(t)-\frac{\lambda_{k} e_{k}(t)}{\left(e_{k}(t) e_{k}(t)\right)^{q}} \\
& \leq-c_{k} e_{k}(t)+\sum_{j=1}^{n} a_{k j} F_{j} e_{j}(t)+\sum_{j=1}^{n} b_{k j} G_{j} e_{j}(t-\tau)-\eta_{k}(t) e_{k}(t)-\frac{\lambda_{k} e_{k}(t)}{\left(e_{k}(t) \bar{e}_{k}(t)\right)^{q}},
\end{aligned}
$$


Then, we choose the following Lyapunov function:

$$
V_{2}(t)=\sum_{k=1}^{n} e_{k}(t) \overline{e_{k}(t)}+\sum_{k=1}^{n}\left(\eta_{k}(t)-\widetilde{\eta}_{k}\right)^{2} .
$$

By using Lemmas 2-5, Equation (16) can be obtained as follows:

$$
\begin{aligned}
& D^{\mu} V_{2}(t) \leq \sum_{k=1}^{n}\left(e_{k}(t) D^{\mu} \overline{e_{k}(t)}+\overline{e_{k}(t)} D^{\mu} e_{k}(t)\right)+D^{\mu} \sum_{k=1}^{n}\left(\eta_{k}(t)-\widetilde{\eta}_{k}\right)^{2} \\
& \leq \sum_{k=1}^{n}\left[\overline{e_{k}(t)}\left(-c_{k} e_{k}(t)+\sum_{j=1}^{n} a_{k j} F_{j} e_{j}(t)+\sum_{j=1}^{n} b_{k j} G_{j} e_{j}(t-\tau)-\eta_{k}(t) e_{k}(t)-\frac{\lambda_{k} e_{k}(t)}{\left(e_{k}(t) e_{k}(t)\right)^{q}}\right)\right. \\
& \left.+e_{k}(t)\left(-\overline{c_{k}} \overline{e_{k}(t)}+\sum_{j=1}^{n} \overline{a_{k j}} \overline{F_{j}} \overline{e_{j}(t)}+\sum_{j=1}^{n} \overline{b_{k j} G_{j}} \overline{e_{j}(t-\tau)}-\overline{\eta_{k}(t) e_{k}(t)}-\frac{\overline{\lambda_{k}} \overline{e_{k}(t)}}{\left(e_{k}(t) \overline{\left.e_{k}(t)\right)^{q}}\right.}\right)\right] \\
& +2 \sum_{k=1}^{n}\left(\eta_{k}(t)-\widetilde{\eta}_{k}\right) D^{\mu} \eta_{k}(t) \\
& \leq \sum_{k=1}^{n}\left[-\left(c_{k}+\overline{c_{k}}\right)+\sum_{j=1}^{n}\left(a_{k j} \overline{a_{k j}} F_{j} \overline{F_{j}}+b_{k j} \overline{b_{k j}} G_{j} \overline{G_{j}}\right)-\left(\eta_{k}(t)+\overline{\eta_{k}(t)}\right)\right] e_{k}(t) \overline{e_{k}(t)} \\
& +\sum_{k, j=1}^{n}\left(e_{j}(t) \overline{e_{j}(t)}+e_{j}(t-\tau) \overline{e_{j}(t-\tau)}\right)-\sum_{k=1}^{n}\left(\lambda_{k}+\overline{\lambda_{k}}\right)\left(e_{k}(t) \overline{e_{k}(t)}\right)^{1-q} \\
& +2 \sum_{k=1}^{n}\left(\eta_{k}(t)-\widetilde{\eta}_{k}\right)\left[e_{k}(t) \overline{e_{k}(t)}-\sigma_{k} \operatorname{sign}\left(\eta_{k}(t)-\widetilde{\eta}_{k}\right)\left|\eta_{k}(t)-\widetilde{\eta}_{k}\right|^{1-2 q}\right] \\
& \leq \sum_{k=1}^{n}\left[-\left(c_{k}+\overline{c_{k}}\right)+\sum_{j=1}^{n}\left(a_{k j} \overline{a_{k j}} F_{j} \overline{F_{j}}+b_{k j} \overline{b_{k j}} G_{j} \overline{G_{j}}\right)-\left(\eta_{k}(t)+\overline{\eta_{k}(t)}\right)\right] e_{k}(t) \overline{e_{k}(t)} \\
& +n \sum_{k=1}^{n}\left(e_{k}(t) \overline{e_{k}(t)}+e_{k}(t-\tau) \overline{e_{k}(t-\tau)}\right)-\sum_{k=1}^{n}\left(\lambda_{k}+\overline{\lambda_{k}}\right)\left(e_{k}(t) \overline{e_{k}(t)}\right)^{1-q} \\
& +2 \sum_{k=1}^{n}\left(\eta_{k}(t)-\widetilde{\eta}_{k}\right)\left[e_{k}(t) \overline{e_{k}(t)}-\sigma_{k} \operatorname{sign}\left(\eta_{k}(t)-\widetilde{\eta}_{k}\right)\left|\eta_{k}(t)-\widetilde{\eta}_{k}\right|^{1-2 q}\right] \\
& \leq \sum_{k=1}^{n}\left[-\left(c_{k}+\bar{c}_{k}+2 \widetilde{\eta}_{k}\right)+\sum_{j=1}^{n}\left(a_{k j} \overline{a_{k j}} F_{j} \overline{F_{j}}+b_{k j} \overline{b_{k j}} G_{j} \overline{G_{j}}\right)+n\right] e_{k}(t) \overline{e_{k}(t)} \\
& +n \sum_{k=1}^{n} e_{k}(t-\tau) \overline{e_{k}(t-\tau)}-\sum_{k=1}^{n}\left(\lambda_{k}+\overline{\lambda_{k}}\right)\left(e_{k}(t) \overline{e_{k}(t)}\right)^{1-q}-2 \sum_{k=1}^{n} \sigma_{k}\left(\left(\eta_{k}(t)-\widetilde{\eta}_{k}\right)^{2}\right)^{1-q}
\end{aligned}
$$

By utilizing Lemma 5, one can get

$$
\begin{aligned}
& \sum_{k=1}^{n}\left(\lambda_{k}+\overline{\lambda_{k}}\right)\left(e_{k}(t) \overline{e_{k}(t)}\right)^{1-q}+2 \sum_{k=1}^{n} \sigma_{k}\left(\left(\eta_{k}(t)-\widetilde{\eta}_{k}\right)^{2}\right)^{1-q} \\
\geq & \min _{1 \leq k \leq n}\left\{\left(\lambda_{i}+\bar{\lambda}_{k}\right), 2 \sigma_{k}\right\} \cdot\left[\sum_{k=1}^{n}\left(e_{k}(t) \overline{e_{k}(t)}\right)^{1-q}+\sum_{k=1}^{n}\left(\left(\eta_{k}(t)-\widetilde{\eta}_{k}\right)^{2}\right)^{1-q}\right] \\
\geq & \widetilde{d}\left[\sum_{k=1}^{n}\left(e_{k}(t) \overline{e_{k}(t)}\right)^{1-q}+\sum_{k=1}^{n}\left(\left(\eta_{k}(t)-\widetilde{\eta}_{k}\right)^{2}\right)^{1-q}\right] \\
\geq & \widetilde{d}\left[\sum_{k=1}^{n} e_{k}(t) \overline{e_{k}(t)}+\sum_{k=1}^{n}\left(\eta_{k}(t)-\widetilde{\eta}_{k}\right)^{2}\right]^{1-q} \\
= & \widetilde{d} V_{2}^{1-q}(t) .
\end{aligned}
$$

In summary, we can deduce

$D^{\mu} V_{2}(t) \leq-Q V_{1}(t)+n V_{1}(t-\tau)-\widetilde{d} V_{2}^{1-q}(t) \leq-\left(Q-n \zeta^{\prime}\right) V_{1}(t)-\widetilde{d} V_{2}^{1-q}(t)$,

for $\zeta^{\prime}>1, \widetilde{d}=\min _{1 \leq k \leq n}\left\{\left(\lambda_{k}+\bar{\lambda}_{k}\right), 2 \sigma_{k}\right\}$, if $Q>n \zeta^{\prime}$, where

$$
Q=\min _{1 \leq k \leq n}\left[\left(c_{k}+\bar{c}_{k}+2 \widetilde{\eta}_{k}\right)-\sum_{j=1}^{n}\left(a_{k j} \overline{a_{k j}} F_{j} \overline{F_{j}}+b_{k j} \overline{b_{k j}} G_{j} \overline{G_{j}}\right)-n\right],
$$


according to Lemma 6, we have

$$
D^{\mu} V_{2}(t) \leq-\widetilde{d} V_{2}^{1-q}(t)
$$

then

$$
V_{2}^{(\mu+q-1)}(t) \leq V_{2}^{(\mu+q-1)}(0)-\frac{\widetilde{d} \Gamma(\mu+q) t^{\mu}}{\Gamma(1+\mu) \Gamma(q)}, 0 \leq t \leq t_{2},
$$

for $t \geq t_{2}, V_{2}(t)=0$, here $t_{2}$ can be estimated by

$$
t_{2}=\left(\frac{\Gamma(1+\mu) \Gamma(q) V_{2}^{\mu+q-1}(0)}{\widetilde{d} \Gamma(\mu+q)}\right)^{\frac{1}{\mu}} .
$$

when $t \geq t_{2}$, we get $\lim _{t \rightarrow \infty}\left\|e_{k}(t)\right\|=0$, then system (8) and system (9) will achieve the finite-time projective synchronization under the adaptive controller (14).

Remark 1. If the projective coefficient $\beta=1$, system (8) and system (9) become complete synchronization.

Remark 2. In the existing literature, the finite-time projective synchronization problems of FOCVNNTD in the complex field have not been investigated, and only the finite-time projective synchronization of fractional-order real-valued systems was considered in [32].

Remark 3. In [14], through the simple feedback controller and adaptive controller, the quasiprojective synchronization and complete synchronization of FOCVNNTD are considered. In [33], in the light of the graph theory method, the criteria for ensuring finite-time synchronization of fractional-order complex-valued coupled systems are obtained. Compared with [33], this paper has designed two new types of feedback controller (11) and adaptive controller (14) to realize the finite-time projective synchronization of FOCVNNs.

Remark 4. Linear feedback controller can restrain the influence of internal characteristics and external disturbances. However, the parameters of the controller are fixed, when the internal characteristics of the system change or the external disturbances change greatly, the performance of the system is often greatly reduced or even unstable. Therefore, it is appropriate to adopt adaptive controller for a class of systems whose object characteristics or disturbance characteristics vary widely, moreover, it is required to keep high performance indexes.

\section{Numerical Simulations}

In this section, there are two numerical simulations are be given to verify the effectiveness and feasibility of the obtained results. The FOCVNNTD are considered as the drive system, the form is as follows:

$$
D^{\mu} x_{k}(t)=-c_{k} x_{k}(t)+\sum_{j=1}^{2} a_{k j} f_{j}\left(x_{j}(t)\right)+\sum_{j=1}^{2} b_{k j} g_{j}\left(x_{j}(t-\tau)\right)+I_{k}, k=1,2,
$$

where $\mu=0.7, x_{j}(t)=z_{j}(t)+i \hat{z}_{j}(t), \tau=1, I(t)=\left(I_{1}(t), I_{2}(t)\right)^{T}=(0,0)$.

$$
\begin{gathered}
f_{j}\left(x_{j}\right)=\frac{1-e^{-z_{j}}}{1+e^{-z_{j}}}+i \frac{1}{1+e^{-z_{j}}}, g_{j}\left(x_{j}\right)=\frac{1-e^{-z_{j}}}{1+e^{-z_{j}}}+i \frac{1}{1+e^{-z_{j}}} . \\
A=\left(a_{k j}\right)_{2 \times 2}=\left(\begin{array}{cc}
3+3 i & -0.2-0.2 i \\
-2-2 i & 1+i
\end{array}\right), \\
C=\operatorname{diag}\left(c_{1}, c_{2}\right)=(1+i, 1+i) \\
B=\left(b_{k j}\right)_{2 \times 2}=\left(\begin{array}{cc}
-0.2-0.2 i & -1.5-1.5 i \\
-0.5-0.5 i & 0.3+0.3 i
\end{array}\right) .
\end{gathered}
$$


The response system is given by:

$$
D^{\mu} y_{k}(t)=-c_{k} y_{k}(t)+\sum_{j=1}^{2} a_{k j} f_{j}\left(y_{j}(t)\right)+\sum_{j=1}^{2} b_{k j} g_{j}\left(y_{j}(t-\tau)\right)+I_{k}+u_{k}(t), k=1,2
$$

For $s \in[-1,0]$, take the initial conditions

$$
\begin{gathered}
x(s)=\left(x_{1}(s), x_{2}(s)\right)=(-0.1+0.2 i, 1+0.3 i), \\
y(s)=\left(y_{1}(s), y_{2}(s)\right)=(0.8+0.5 i, 0.9-0.6 i) .
\end{gathered}
$$

For convenience of calculation, choose $F_{j}=G_{j}=\sqrt{2}$.

Figure 1 shows the phase trajectories of the real and imaginary parts of the error system (10).

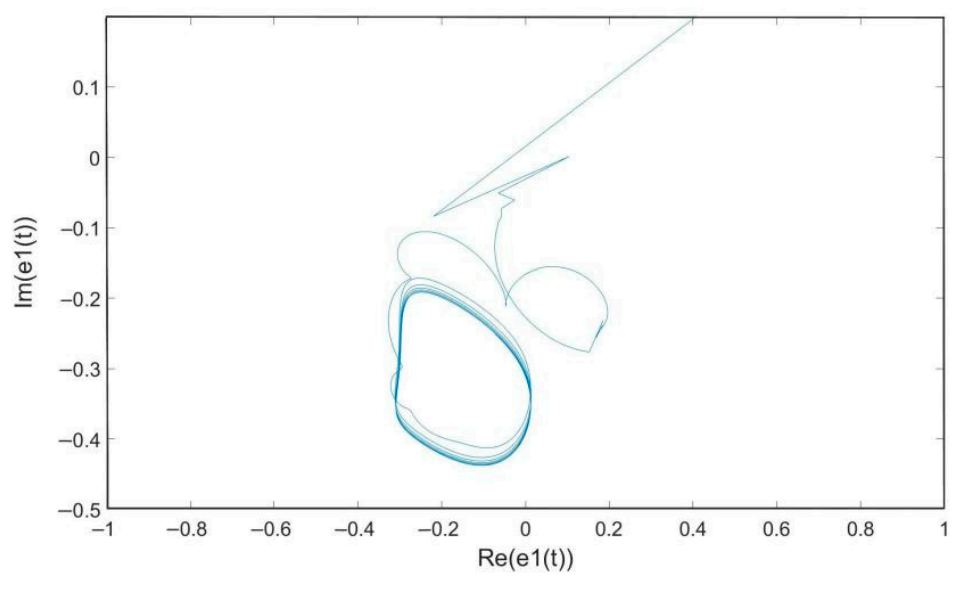

(a)

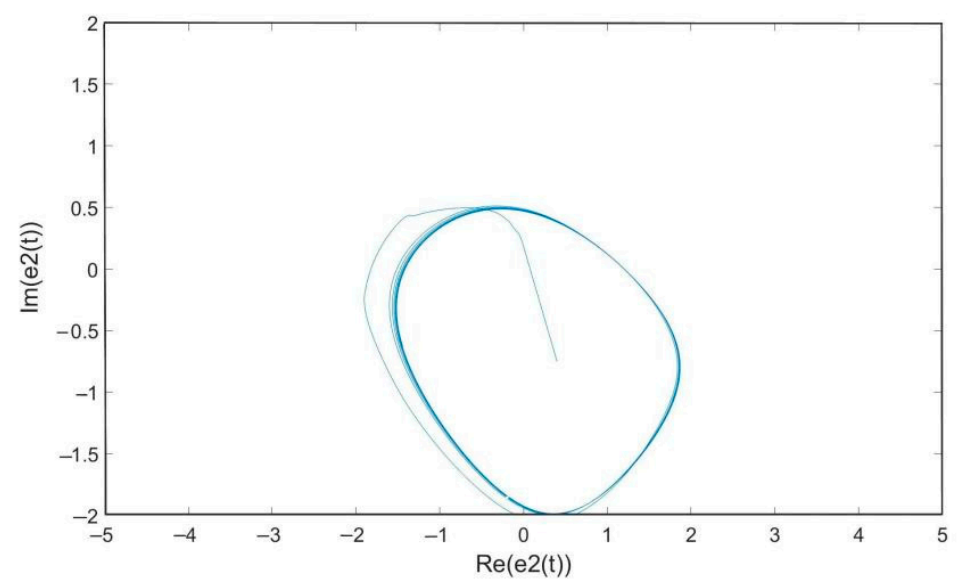

(b)

Figure 1. (a) The phase trajectories of real and imaginary part of system (10). (b) The phase trajectories of real and imaginary part of system (10).

Example 1. In order to realize the finite-time projective synchronization of the drive system (19) and the response system (20), the system (11) is designed as a linear feedback controller, where

$$
q=0.1, \text { take }_{1}=30+30 i, \eta_{2}=35+35 i, \zeta=1.5, \lambda=\left(\lambda_{1}, \lambda_{2}\right)=(2.5+2.5 i, 3+3 i) .
$$


By simple calculation,

$$
P=\min _{1 \leq k \leq 2}\left[\left(c_{k}+\overline{c_{k}}+\eta_{k}+\overline{\eta_{k}}\right)-\sum_{j=1}^{2}\left(a_{k j} \overline{a_{k j}} F_{j} \overline{F_{j}}+b_{k j} \overline{b_{k j}} G_{j} \overline{G_{j}}\right)-n\right]=14.68,
$$

$n \zeta=3<P$. Therefore, the conditions and assumptions are satisfied in Theorem 1 , and the obtained settling time is $t_{1} \approx 1.1678$.

The state trajectories of the real and imaginary parts of the error system (10) and error norm trajectory curve are depicted in Figure 2 when $\beta=0.6+0.5 i$, which shows that the system (19) and the system (20) can reach the finite-time projective synchronization under the feedback controller (11). Figure 3 displays the state trajectories of the real and imaginary parts of the error system (10) and error norm trajectory curve when $\beta=1$, which shows that the system (19) and the system (20) will accomplish the complete synchronization under the feedback controller (11).

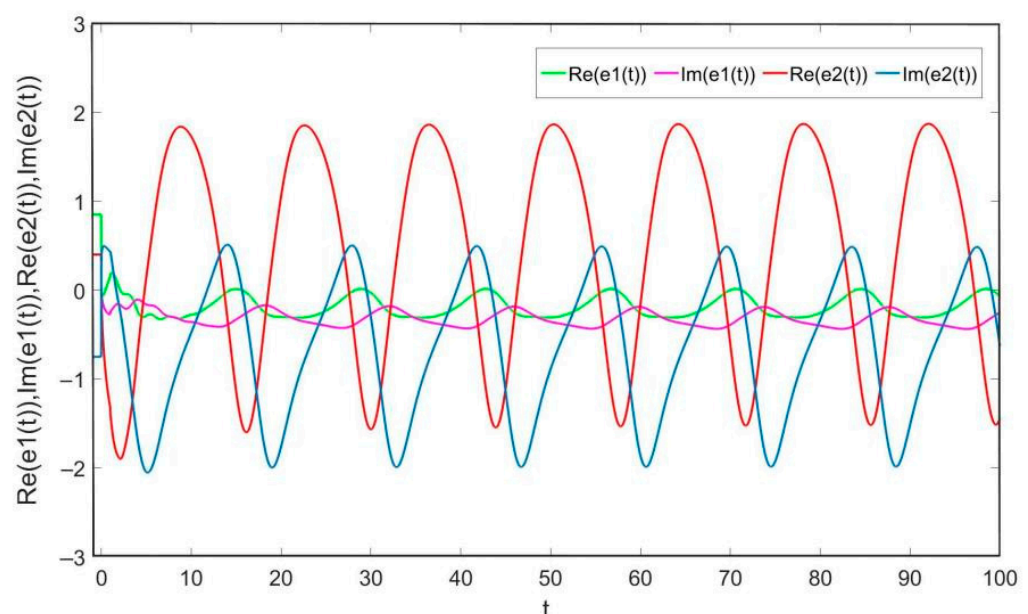

(a)

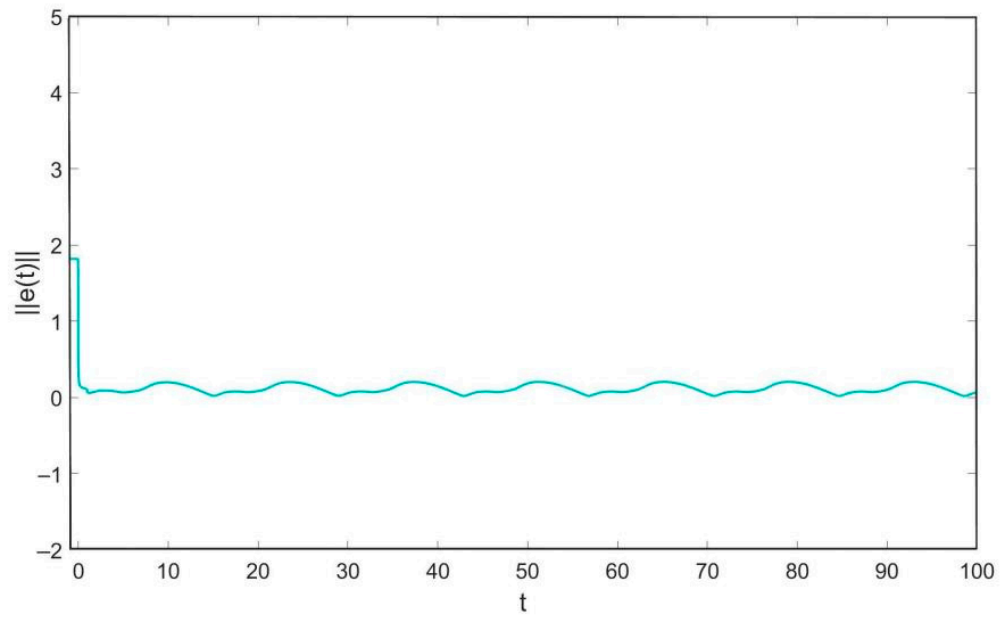

(b)

Figure 2. (a) The state trajectory of the error system when $\beta=0.6+0.5 i$. (b) The trajectory of the error norm $\beta=0.6+0.5 i$. 


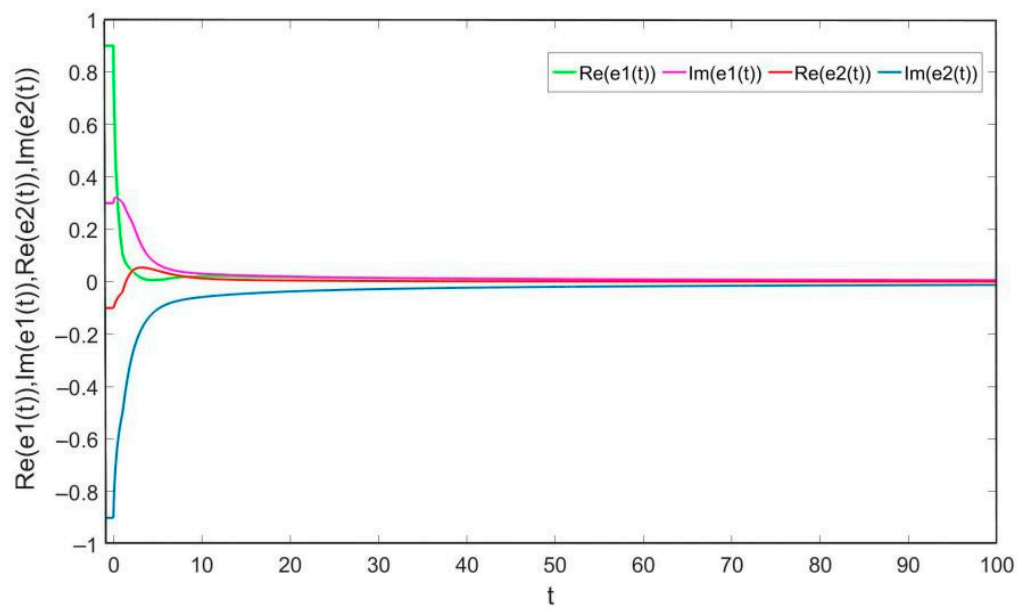

(a)

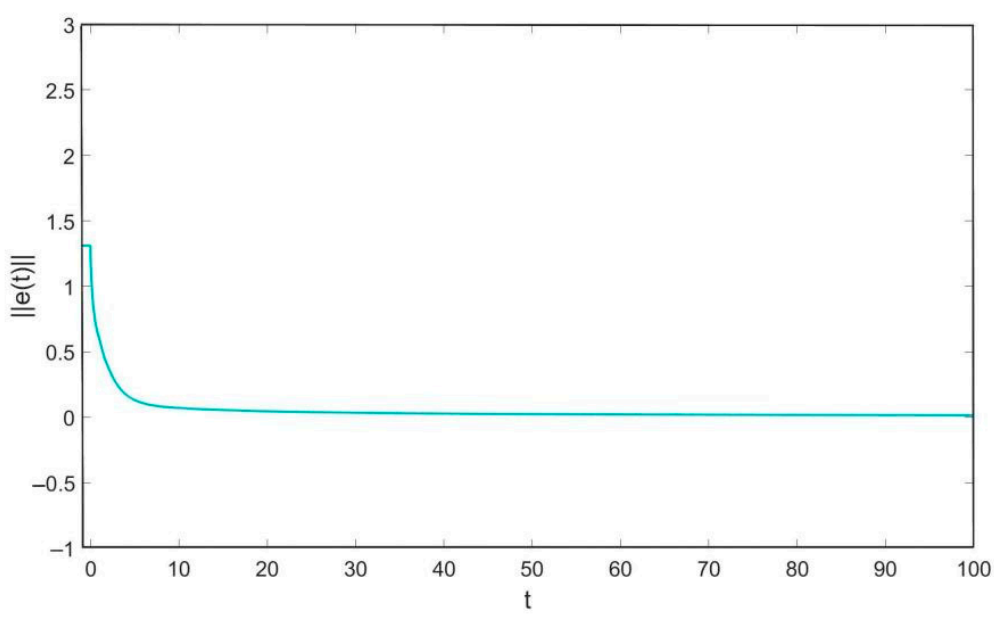

(b)

Figure 3. (a) The state trajectory of the error system when $\beta=1$. (b). The trajectory of the error norm $\beta=1$.

Example 2. In order to realize the finite-time projective synchronization of the system (19) and the system (20), the system (14) is built as an adaptive controller, where $q=0.4, \zeta^{\prime}=1.8, \sigma_{1}=0.3$, $\sigma_{2}=0.6, \widetilde{\eta}_{1}=25, \widetilde{\eta}_{2}=20, \eta_{1}(0)=0.1, \eta_{2}(0)=0.2, \lambda=\left(\lambda_{1}, \lambda_{2}\right)=(2.5+2.5 i, 3+3 i) . B y$ simple calculation, we have

$$
Q=\min _{1 \leq k \leq 2}\left[\left(c_{k}+\bar{c}_{k}+2 \widetilde{\eta}_{k}\right)-\sum_{j=1}^{n}\left(a_{k j} \overline{a_{k j}} F_{j} \overline{F_{j}}+b_{k j} \overline{b_{k j}} G_{j} \overline{G_{j}}\right)-n\right]=4.68,
$$

$n \zeta^{\prime}=3.6<Q$. Similarly, the conditions and assumptions are satisfied in Theorem 2 , and the obtained settling time is $t_{2} \approx 1.6169$. The state trajectories of the real and imaginary parts of the error system (10) and error norm trajectory curve are showed in Figure 4 when $\beta=0.6+0.5 i$, which shows that the system (19) and the system (20) will achieve the finite-time projective synchronization under the adaptive controller (14). Figure 5 depicts the state trajectories of the real and imaginary parts of the error system (10) and error norm trajectory curve when $\beta=1$, which shows that the system (19) and the system (20) will achieve the complete synchronization under the adaptive controller (14). 


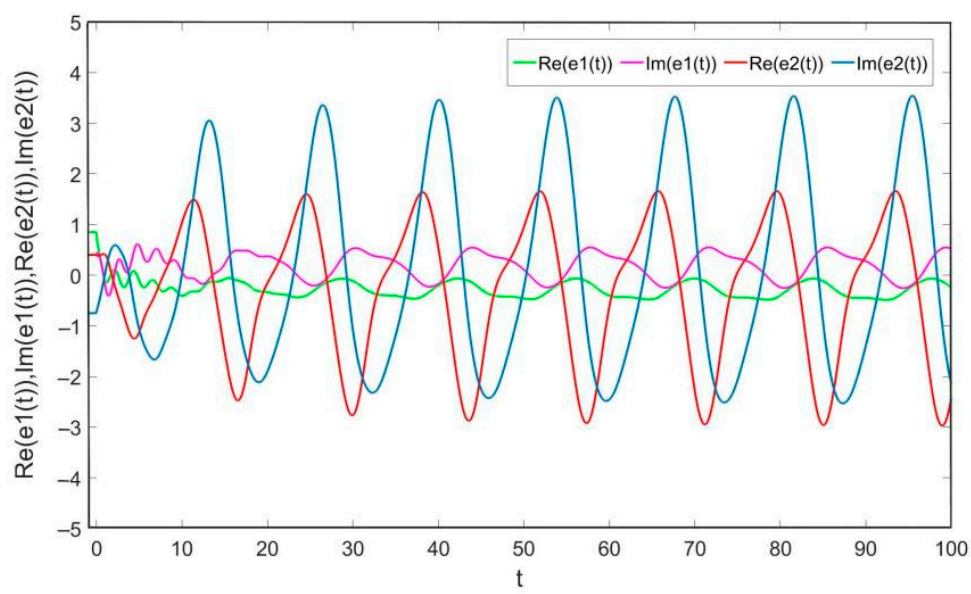

(a)

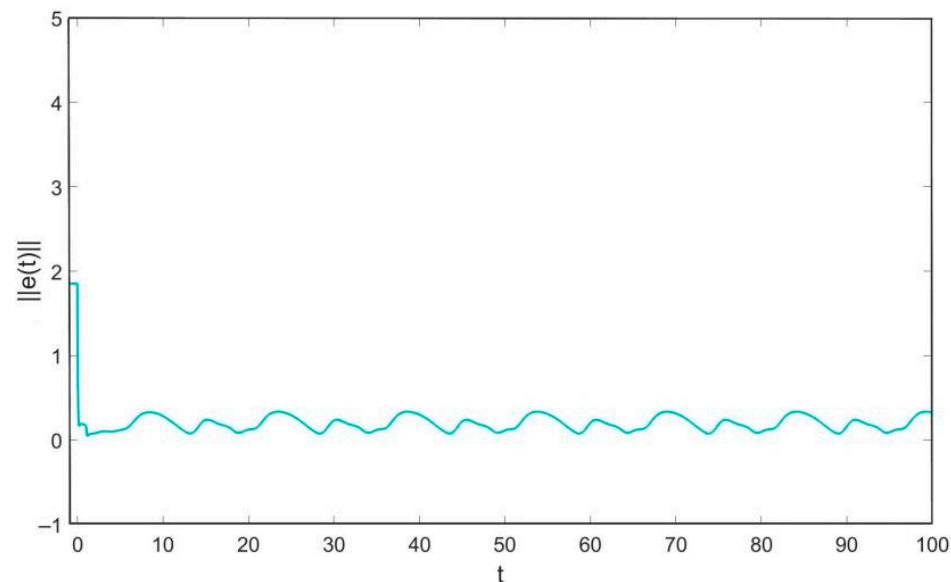

(b)

Figure 4. (a) The state trajectory of the error system when $\beta=0.6+0.5 i$. (b) The trajectory of the error norm $\beta=0.6+0.5 i$.

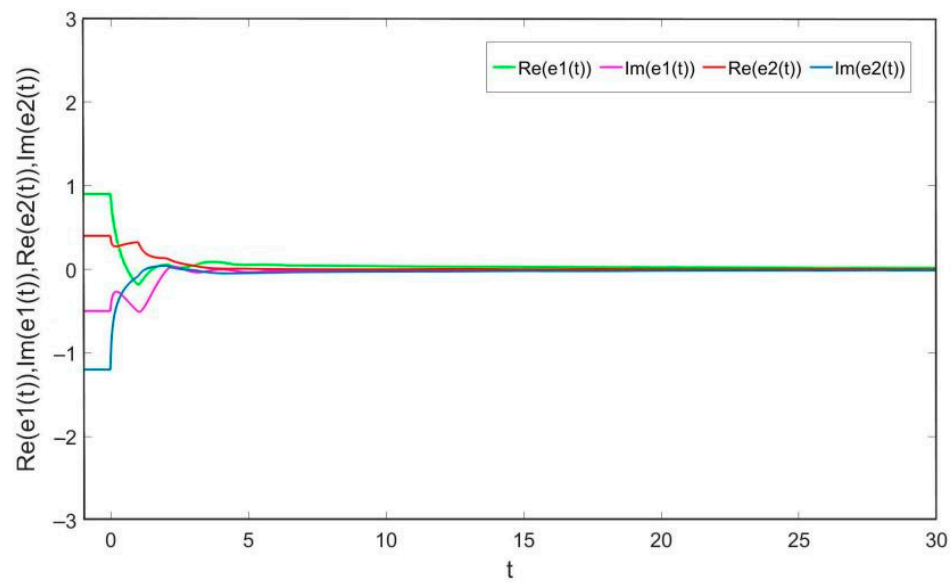

(a)

Figure 5. Cont. 


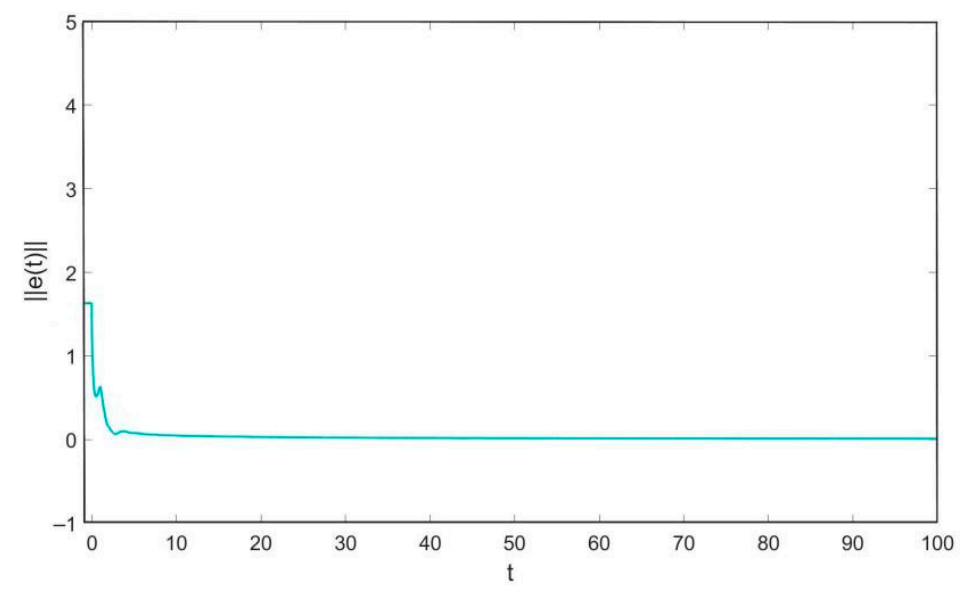

(b)

Figure 5. (a) The state trajectory of the error system when $\beta=1$. (b) The trajectory of the error norm $\beta=1$.

\section{Conclusions}

In this paper, a feedback controller and adaptive controller were designed to study the finite-time projective synchronization problem of the FOCVNNTD. Some different criteria for ensuring the finite-time projective synchronization of the FOCVNNTD were established. Furthermore, the synchronization time was estimated effectively. Finally, some numerical simulations are given to illustrate the effectiveness and applicability of the obtained results.

Author Contributions: Formal analysis, H.Z. (Hongmei Zhang); Project administration, H.Z. (Hai Zhang); Software, W.Z.; Supervision, H.Z. (Hai Zhang); Writing-original draft, S.W. All authors contributed equally to this article. All authors have read and agreed to the published version of the manuscript.

Funding: This work is jointly supported by the Natural Science Foundation of Anhui Province (No. 1908085MA01), the Natural Science Foundation of the Higher Education Institutions of Anhui Province (No. KJ2019A0573, No. KJ2019A0557) and the Top Young Talents Program of Higher Learning Institutions of Anhui Province (No. gxyq2019048).

Institutional Review Board Statement: Not applicable.

Informed Consent Statement: Not applicable.

Data Availability Statement: All data generated during this study are included in this article, and the codes are available from the corresponding author on reasonable request.

Conflicts of Interest: The authors declare that they have no conflict of interest.

\section{References}

1. Zhang, T.T.; Yu, Y.G.; Cui, X.L. Dynamical behaviors analysis of memristor-based fractional-order complex-valued neural networks with time delay. Appl. Math. Comput. 2018, 339, 242-258. [CrossRef]

2. Zhang, W.W.; Zhang, H.; Cao, J.D.; Zhang, H.M.; Chen, D.Y. Synchronization of delayed fractional-order complex-valued neural networks with leakage delay. Phys. A Stat. Mech. Its Appl. 2020, 556, 124710. [CrossRef]

3. Zhang, H.; Ye, M.; Ye, R.; Cao, J. Synchronization stability of Riemann-Liouville fractional delay-coupled complex neural networks. Phys. A Stat. Mech. Its Appl. 2018, 508, 155-165. [CrossRef]

4. Hu, T.T.; He, Z.; Zhang, X.J.; Zhong, S.M. Finite-time stability for fractional-order complex-valued neural networks with time delay. Appl. Math. Comput. 2020, 365, 124715. [CrossRef]

5. Zheng, B.B.; Hu, C.; Yu, J.; Jiang, H.J. Finite-time synchronization of fully complex-valued neural networks with fractional-order. Neurocomputing 2020, 373, 70-80. [CrossRef]

6. Zhang, H.; Ye, R.; Cao, J.; Alsaedi, A. Delay-independent stability of Riemann-Liouville fractional neutral-type delayed neural networks. Neural Process. Lett. 2018, 47, 427-442. [CrossRef]

7. Alimi, A.M.; Aouiti, C.; Assali, E.A. Finite-time and fixed-time synchronization of a class of inertial neural networks with multi-proportional delays and its application to secure communication. Neurocomputing 2019, 332, 29-43. [CrossRef] 
8. Wen, S.; Zeng, Z.; Huang, T.; Meng, Q.; Yao, W. Lag synchronization of switched neural networks via neural activation function and applications in image Encryption. Neural Netw. 2015, 26, 1493-1502. [CrossRef]

9. Hoppensteadt, F.; Izhikevich, E. Pattern recognition via synchronization in phase-locked loop neural networks. IEEE Trans. Neural Netw. 2000, 11, 734-738. [CrossRef]

10. Gan, Q.T.; Xu, R.; Kang, X.B. Synchronization of chaotic neural networks with mixed time-delays. Commun. Nonlinear Sci. Numer. Simul. 2011, 16, 966-974. [CrossRef]

11. Zhang, W.; Cao, J.; Wu, R.; Alsaadi, F.E.; Alsaedi, A. Lag projective synchronization of fractional-order delayed chaotic systems. J. Frankl. Inst. 2019, 356, 1522-1534. [CrossRef]

12. Wei, X.; Zhang, Z.; Liu, M.; Wang, Z.; Chen, J. Anti-synchronization for complex-valued neural networks with leakage delay and time-varying delays. Neurocomputing 2020, 412, 312-319. [CrossRef]

13. Yang, X.; Li, C.; Huang, T.; Song, Q.; Chen, X. Quasi-uniform synchronization of fractional-order memristor-based neural networks with delay. Neurocomputing 2017, 234, 205-215. [CrossRef]

14. Li, H.L.; Hu, C.; Cao, J.D.; Jiang, H.J.; Alsaedi, A. Quasi-projective and complete synchronization of fractional-order complexvalued neural networks with time delays. Neural Netw. 2019, 118, 102-109. [CrossRef] [PubMed]

15. Zhang, W.W.; Zhang, H.; Cao, J.D.; Alsaadi, F.E.; Chen, D.Y. Synchronization in uncertain fractional-order memristive complexvalued neural networks with multiple time delays. Neural Netw. 2019, 110, 186-198. [CrossRef]

16. Chen, J.J.; Zeng, Z.G.; Jiang, P. Global Mittag-Leffler stability and synchronization of memristor-based fractional-order neural networks. Neural Netw. 2014, 51, 1-8. [CrossRef] [PubMed]

17. You, X.X.; Song, Q.K.; Zhao, Z.J. Global Mittag-Leffler stability and synchronization of discrete-time fractional-order complexvalued neural networks with time delay. Neural Netw. 2020, 122, 382-394. [CrossRef] [PubMed]

18. Zhang, H.; Xie, Y.; Wang, Z.; Zheng, C. Adaptive synchronization between two different chaotic neural networks with time delay. IEEE Trans. Neural Netw. 2007, 18, 1841-1845. [CrossRef]

19. Xu, W.; Zhu, S.; Fang, X.Y.; Wang, W. Adaptive synchronization of memristor-based complex-valued neural networks with time delays. Neurocomputing 2019, 364, 119-128. [CrossRef]

20. Li, H.L.; Jiang, Y.L.; Wang, Z.L. Anti-synchronization and intermittent anti-synchronization of two identical hyperchaotic Chua systems via impulsive control. Nonlinear Dyn. 2015, 79, 919-925. [CrossRef]

21. Zhang, G.D.; Shen, Y. Exponential synchronization of delayed memristor-based chaotic neural networks via periodically intermittent control. Neural Netw. 2014, 55, 1-10. [CrossRef]

22. Velmurugan, G.; Rakkiyappan, R.; Cao, J.D. Finite-time synchronization of fractional-order memristor-based neural networks with time delays. Neural Networks 2016, 73, 36-46. [CrossRef] [PubMed]

23. Du, F.F.; Lu, J.G. New criterion for finite-time synchronization of fractional-order memristor-based neural networks with time delay. Appl. Math. Comput. 2021, 389, 125616. [CrossRef]

24. Kilbas, A.A.; Srivastava, H.M.; Trujillo, J.J. Theory and Application of Fractional Differential Equations; Elsevier Science B.V.: Amsterdam, The Netherlands, 2006.

25. Li, C.; Deng, W. Remarks on fractional derivatives. Appl. Math. Comput. 2007, 187, 777-784. [CrossRef]

26. Yang, S.; Yu, J.; Hu, C.; Jiang, H.J. Quasi-projection synchronization of fractional-order complex-valued recurrent neural networks. Neural Netw. 2018, 104, 104-112. [CrossRef]

27. Chen, Z.; Wu, G.; Fu, X. Synchronization of a network coupled with complex-variable chaotic systems. Chaos $2012,22,023127$.

28. Yu, J.; Hu, C.; Jiang, H. Corrogendum to projective synchronization for fractional neural networks. Neural Netw. 2015, 67, 152-154. [CrossRef]

29. Kuczma, M. An Introduction to the Theory of Functional Equations and Inequalities: Cauchy's Equation and Jensen's Inequality; Birkhauser Verlag AG: Basel, Switzerland; Boston, MA, USA; Berlin, Germany, 2009.

30. Li, H.L.; Cao, J.; Jiang, H.; Alsaedi, A. Graph theory-based finite-time synchronization of fractional-order complex dynamical networks. J. Frankl. Inst. 2018, 355, 5771-5789. [CrossRef]

31. Qin, X.; Wang, C.; Li, L. Finite-time modified projective synchronization of memristor-based neural networks with multi-links and leakage. Chaos Solitons Fractals 2018, 116, 302-315. [CrossRef]

32. Qin, X.; Wang, C.; Li, L.; Peng, H.; Yang, Y.; Ye, L. Finite-time projective synchronization of memristor-based neural networks with leakage and time-varying delays. Phys. A Stat. Mech. Its Appl. 2019, 531, 121788. [CrossRef]

33. Xu, Y.; Li, W.X. Finite-time synchronization of fractional-order complex-valued coupled systems. Phys. A Stat. Mech. Its Appl. 2020, 549, 123903. [CrossRef] 